\title{
Review on Design and Measurement of BER of faded QPSK modulated system
}

\author{
Arvinder Singh \\ Department of Computer Science Engineering \\ ASRA College of Engineering \&Technology, Bhawanigarh, India \\ Rajnish Kansal \\ Faculty of Computer Science Engineering Department \\ ASRA College of Engineering \&Technology, Bhawanigarh, India
}

\begin{abstract}
The usage of the wireless spectrum has been given much research focus due to its limited capacity. Wireless Communication is an application of science and technology that has come to be vital for modern existence. From the early radio and telephone to current devices such as mobile phones and laptops, accessing the global network has become the most essential and indispensable part of our lifestyle. Wireless communication is an ever-developing field, and the future holds many possibilities in this area. Deployment of Wireless LAN increases well around the globe, it is increasingly important for us to understand different technologies and select the most appropriate one. MIMO channel also has been implemented and the throughput of the system has been calculated. As it known, wireless channels key problem is fading, in order to combat this fading and improve the capacity and the throughput of the system, multiple antennas at both ends of the communication link are used. This paper gives the review of Design and Measurement of BER of faded QPSK modulated system.
\end{abstract}

Keywords - QPSK, BER, Wireless, LAN.

\section{INTRODUCTION}

Wireless [7] is a medium in which electromagnetic waves carry the signal over part or the entire communication path. The term is usually used in the telecommunications industry to refer to telecommunications systems (e.g. radio transmitters and receivers, remote controls etc.) which use some form of energy to transfer information without the use of wires. This implies the transfer of information between two or more points that are not connected by an electrical conductor. Some wireless technologies use electromagnetic wireless telecommunications, such as radio. It consists of various types of fixed, mobile, and portable applications, including cellular telephones, personal digital assistants (PDAs). It can be divided into:

- Wireless at fixed location - The operation of wireless devices or systems in homes and offices, and in particular, equipment connected to the Internet via modems.

- Wireless in mobile applications - The use of wireless devices or systems on high speed moving vehicles like automotive cell phone and PCS (personal communications services)

- Wireless for portable applications - The operation of handheld, battery-powered wireless devices or systems outside the premises or vehicle come under this category.

- Infrared wireless - The use of devices that convey data via IR (infrared) radiation is called Infrared wireless. This is employed in certain limited-range communications and control systems.

The increased demands for mobility and flexibility in our daily life are demands that lead the development from wired LANs to wireless LANs (WLANs) [13]. Today a wired LAN can offer users high bit rates to meet the requirements of bandwidth consuming services like video conferences, streaming video etc. With this in mind a user of a WLAN will have high demands on the system and will not accept too much degradation in performance to achieve mobility and flexibility. This will in turn put high demands on the design of WLANs of the future. A wireless LAN is based on a cellular architecture where the system is subdivided into cells, where each cell (called Base Service Set or BSS) is controlled by a Base station (called Access point or AP).

\section{LITERATURE REVIEW}


Mico, $\mathrm{F}$ et al. [8] in 2004 discussed that the IEEE 802.11 wireless LAN (WLAN) is the most widely used standard nowadays for wireless LAN technology. However, the current standard does not provide the QoS support required by the wide range of end applications. Thus, a large number of enhancements to the standard are being proposed. The paper presents a survey of the on-going research activities. They also overviewed the upcoming IEEE 802.11e standard being introduced. The IEEE 802.11e standard is a proposal defining the mechanisms for wireless LANs aiming to provide QoS support to bandwidth-sensitive applications such as voice and video communications.

Le Chung Tran et al. [6] in 2005 discussed that although generation of correlated Rayleigh fading envelopes has been intensively considered in the literature, all conventional methods have their own shortcomings, which seriously impede their applicability. A very general, straight forward algorithm for the generation of an arbitrary number of Rayleigh envelopes with any desired, equal or unequal power, in wireless channels either with or without Doppler frequency shifts, is proposed. The proposed algorithm can be applied to the case of spatial correlation, such as with multiple antennas in multiple-input multiple-output (MIMO) systems, or spectral correlation between the random processes like in orthogonal frequency-division multiplexing (OFDM) systems. It can also be used for generating correlated Rayleigh fading envelopes in either discrete-time instants or a real-time scenario. Besides being more generalized, our proposed algorithm is more precise, while overcoming all shortcomings of the conventional methods.

Ahmed M. Al Naamany et al. [2] in 2006 discussed that the Wireless Local Area Networks (WLANs) are cost effective and desirable gateways to mobile computing. They allow computers to be mobile, cable less and communicate with speeds close to the speeds of wired LANs. These features came with expensive price to pay in areas of security of the network. This paper identifies and summarizes these security concerns and their solutions. Broadly, security concerns in the WLAN world are classified into physical and logical. The paper overviews both physical and logical WLANs security problems followed by a review of the main technologies used to overcome them. It addresses logical security attacks like manin-the-middle attack and Denial of Service attacks as well as physical security attacks like rouge APs. Wired Equivalent Privacy (WEP) was the first logical solution to secure WLANs. However, WEP suffered many problems which were partially solved by IEEE $802.1 \mathrm{x}$ protocol. Towards perfection in securing WLANs, IEEE802.11i emerged as a new MAC layer standard which permanently fixes most of the security problems found in WEP and other temporary WLANs security solutions. This paper reviews all security solutions starting from WEP to IEEE802.11i and discusses the strength and weakness of these solutions.

Shanar H. Askar [11] in 2010 discussed that a radio channel characteristic modeling is essential in every network planning. This deals with the performance of WiMax networks in an outdoor environment while using fading channel models. The radio channels characteristics are analyzed by simulations have been done using Matlab programming. Stanford University Interim (SUI) Channels set was proposed to simulate the fixed broadband wireless access channel environments where IEEE 802.16d is to be deployed. It has six channel models that are grouped into three categories according to three typical different outdoor Terrains, in order to give a comprehensive study of fading channels on the overall performance of the system, WiMax system has been tested under SUI channels that modified into account for 300 directional antennas, with $90 \%$ cell coverage and with $99.9 \%$ reliability in its geographical covered area. Furthermore, in order to combat the fading which occurs in urban areas and improve the capacity and the throughput of the system, multiples antennas at both ends of communication link are used, the transmission gain obtained when using multiple antennas instead of only a single antenna. Space-time coding and maximum ratio combining for more than one transmit and receive antenna is implemented to allow performance investigations in various MIMO scenarios. It has been concluded that uses multiple antennas at the receiver offers a significant improvement of $3 \mathrm{~dB}$ of gain in the channel SNR.

R K Bansal et al.[9] in 2010 discussed that the wired Computer Networks provide a secure and faster means of connectivity but the need of mobility i.e. anywhere, anytime and anyone access is tilting the network users towards wireless technology. In this paper, an overview of the current research literature, in the field of Wired and wireless networks, has been presented. The network simulators provide an ease in predicting and estimating the performance of networks. Among the various network simulators available, OPNET gains an edge in analysing the performance of the networks through simulations. The metrics like throughput, delay and retransmission attempts have been overviewed for performance analysis of the wireless and wired computer networks using soft computing techniques like simulation through OPNET.

Rahul P. Bhoyar et al. [10] in 2013 discussed that wireless Communication is an application of science and technology that has come to be vital for modern existence. From the early radio and telephone to current devices such as mobile phones and laptops, accessing the global network has become the most essential and indispensable part of our lifestyle. Wireless Local Area Network is a data transmission system considered to provide location independent network access between computing devices by using radio waves rather than a cable infrastructure. In the business venture, wireless LANs are frequently employed as the final link between the existing wired network and a group of client computers, giving these users wireless access to the full resources and services of the corporate network across 
a building or campus setting. The 802.11 is a family of specifications developed by the IEEE for WLANs. The IEEE 802.11 standard supports radio transmission within the $2.4 \mathrm{GHz}$ band. In this paper, we concern on different WLAN standards and their comparative study on standard $802.11 \mathrm{a} / \mathrm{b} / \mathrm{g} / \mathrm{h}$ which will help to WLAN user's, student and researcher's for selection of better type of WLAN standard.

Keerti Tiwari et al.[5] in 2014 discussed that in the present wireless scenario, multiple-input and multiple-output (MIMO) is an emerging technique in wireless communication to achieve reliability and high throughput. Transmit diversity i.e. Alamouti space time block codes (STBC) and receiver diversity i.e. maximal ratio combining (MRC), are used to improve the link performance. In this paper, bit error rate (BER) performance is evaluated using distinct modulation schemes like BPSK, QPSK, and 16-QAM with STBC and MRC diversity techniques over Rayleigh, Rician and Nakagami-m fading channels.

Xiaohui Wang [14] in 2014 discussed that wireless research and development requires effective and efficient simulation and emulation tools to validate and evaluate wireless designs. Wireless channel models are used in the tools to simulate signal propagation properties in the real physical world. However, due to practical issues, these models are often too generalized and simplified in large scale experiments, and they only provide limited realism. In this, a novel world model is proposed for simulation and emulation of wireless networks. The proposed model includes the design and implementation of a variety of environment models that enhance realism in simulation. These models capture realistic signal propagation properties across multiple connections, and over time: first, the impact of realistic physical world features, such as channel dynamics and cross link correlation are characterized at different time scales; then, both geometrical and statistical simulation models are developed to recreate desired channel dynamics among wireless network links efficiently.

Arashpal Chahal et al. [3] in 2015 discussed that increasing demand for high-performance 4G broadband wireless is enabled by the use of multiple antennas at both base station and subscriber ends. Multiple antenna technologies enable high capacities suited for Internet and multimedia services, and also dramatically increase range and reliability. However, in fast fading channels, the time variation of a fading channel over OFDM symbol period results in a loss of sub-channel orthogonality, which leads to inter-carrier interference (ICI). In this paper, introduce an orthogonal spatial division multiplexing in which divide the central signal streams into both time and frequency. Also to increase the spatial diversity we are going to introduce spatial modulation along with STBC for our new MIMO-OSDM. Experimental results show that, proposed system outperform the existing MIMO-OFDM system in terms of bit and symbol error rate for various modulation schemes.

Abu Taha Zamani [1] et al. discussed that 802.11g is an exciting new technology that offers additional performance, while providing investment protection for $802.11 \mathrm{~b}$ clients through backward compatibility. By using previous technologies and economies of scale, $802.11 \mathrm{~g}$ devices are available at little or no additional cost relative to 802.11b. As such, there are many reasons to begin migrating from 802.11b to 802.11g.802.11g is not, however, a panacea for WLAN capacity issues. As WLAN capacity needs increase, network professionals are well-advised to begin deploying a dual-band infrastructure to access the far greater capacity available with 802.11a. As such, 802.11g should be viewed as a portion of an overall WLAN architecture, not a substitute for 802.11a. 802.11g is a "bridge" technology and an ideal means for migrating from low-capacity $802.11 \mathrm{~b}$ networks to the high-capacity, dual-band WLANs of the very near future. The evolution of wireless networking in recent years has raised many serious security issues. These security issues are of great concern for this technology as it is being subjected to numerous attacks. Because of the free-space radio transmission in wireless networks, eavesdropping becomes easy and consequently a security breach may result in unauthorized access, information theft, interference and service degradation. Virtual Private Networks (VPNs) have emerged as an important solution to security threats surrounding the use of public networks for private communications. This paper presents an approach to secure IEEE 802.11g WLAN using Open VPN, a transport layer VPN solution and its impact on performance of IEEE 802.11g WLAN.

Bachir Habib et al. [4] discussed that a wireless communication system can be tested either in actual conditions or by using a hardware simulator reproducing actual conditions. With a hardware simulator it is possible to freely simulate a desired type of a radio channel. This paper presents architectures for the digital block of a hardware simulator of Multiple-Input Multiple-Output (MIMO) propagation channels. This simulator can be used for Long Term Evolution System (LTE) and Wireless Local Area Networks (WLAN) 802.11ac applications, in indoor and outdoor environments. The first architecture is appropriate for shipboard environments, while the second corresponds to outdoor-to-indoor environments and considers the wave propagation penetration within buildings. Measurements campaigns carried out at 2.2 and $3.5 \mathrm{GHz}$ have been conducted to obtain the impulse responses of the channel using a MIMO channel sounder designed at IETR. The measurements are processed with an algorithm extracting the dominant paths. The architectures of the digital block are implemented on a Xilinx Virtex-IV Field Programmable Gate Array (FPGA). After the implementation of the impulse responses, the accuracy, the occupation on the FPGA and the latency of the architectures are analyzed. 
Vijay Chandramouli [12] discussed that wireless Communication is an application of science and technology that has come to be vital for modern existence. From the early radio and telephone to current devices such as mobile phones and laptops, accessing the global network has become the most essential and indispensable part of our lifestyle. Wireless communication is an ever-developing field, and the future holds many possibilities in this area. One expectation for the future in this field is that, the devices can be developed to support communication with higher data rates and more security. Research in this area suggests that a dominant means of supporting such communication capabilities will be through the use of Wireless LANs. As the deployment of Wireless LAN increases well around the globe, it is increasingly important for us to understand different technologies and select the most appropriate one. This paper provides a detailed study of the available wireless LAN technologies and the concerned issues. This is followed by a discussion evaluating and suggesting a feasible standard for future.

\section{IV.CONCLUSION}

Wireless communication is an ever-developing field, and the future holds many possibilities in this area. Deployment of Wireless LAN increases well around the globe, it is increasingly important for us to understand different technologies and select the most appropriate one. MIMO channel also has been implemented and the system BER has been calculated. As it known, wireless channels key problem is fading, in order to combat this fading and improve the capacity and the signal to noise ratio of the system, multiple antennas at both ends of the communication link are used. This paper reviewed the performance analysis of MIMO in Rayleigh fading envelope.

\section{REFERENCES}

[1] Abu Taha Zamani and Javed Ahmad (2014), "Wireless LAN Security : IEEE 802.11g \& VPN", International Journal of Advanced Research in Computer Science and Software Engineering, Volume 4, Issue 2.

[2] Ahmed M. Al Naamany, Ali Al Shidhani, Hadj Bourdoucen(2006), "IEEE 802.11 Wireless LAN Security Overview", IJCSNS International Journal of Computer Science and Network Security, Vol.6 No.5B.

[3] Arashpal Chahal, Amandeep Kaur and Ranjeet Singh(2015), "An Adaptive MIMO-OSDM Prototype for Next Generation Communication Systems under fading Channels", International Journal of Engineering Research and General Science, Volume 3, Issue 1.

[4] Bachir Habib, Gheorghe Zaharia, Hanna Farhat and Ghais El Zein(2013), “MIMO Hardware Simulator Using Standard Channel Models and Measurement Data at 2.2 and 3.5 GHz", Journal of Communication and Computer, 10 (4), pp.525-544.

[5] Keerti Tiwari and Davinder S Saini(2014), "BER Performance Comparison ofMIMO System with STBC and MRC over Different Fading Channels", IEEE.

[6] Le Chung Tran, Tadeusz A. Wysocki, Alfred Mertins and Jennifer Seberry (2005), "A Generalized Algorithm for the Generation of Correlated Rayleigh Fading Envelopes in Wireless Channels", EURASIP Journal on Wireless Communications and Networking, 801-815.

[7] Ma Yue, J. James Han, S. Trivedi., Kishor "Composite Performance and Availability Analysis of Wireless Communication Networks," IEEE Transactions on Vehicular Technology, vol. 50, no. 5, pp.1216-1223, 2001.

[8] Mico, F., Cuenca, P. and Orozco-Barbosa, L.(2004), "QoS in IEEE 802.11 wireless LAN:current research activities", Electrical and Computer Engineering, Canadian Conference,IEEE, pp. 447 - 452 Vol.1.

[9] R K Bansal, Vikas Gupta and Rahul Malhotra (2010), "Performance Analysis of Wired and Wireless LAN Using Soft Computing Techniques- A Review", Global Journal of Computer Science and Technology Vol. 10 Issue 8 Ver.1.0.

[10] Rahul P. Bhoyar, Mangesh M. Ghonge and Suraj G. Gupta(2013), "Comparative Study on IEEE Standard of Wireless LAN/ Wi-Fi 802.11 $\mathrm{a} / \mathrm{b} / \mathrm{g} / \mathrm{n}$ ", International Journal of Advanced Research in Electronics and Communication Engineering (IJARECE), Volume 2, Issue 7.

[11] Shanar H. Askar(2010), "Performance Evaluation of Fixed WiMax Physical Layer under High Fading Channels".

[12] Vijay Chandramouli, "A Detailed Study on Wireless LAN Technologies".

[13] Won-Gyu Song; Jong-Tae Lim, "Pilot-symbol aided channel estimation for OFDM with fast fading channels,", IEEE Transactions on Broadcasting, vol.49, no.4, pp.398,402, Dec. 2003.

[14] Xiaohui Wang (2014), "Environment Models for Realistic Simulation and Emulation of Wireless Networks". 